\title{
Retroperitoneal lymphatic malformation: a case report
}

\begin{abstract}
Lymphatic malformations are rare congenital vascular malformations with head and neck are the most usual location. Less commonly seen in the retroperitoneam with cases compromised less than $1 \%$ from all abdominal cases. Majority of the patients are asymptomatic, however minority may present with abdominal distension or pain, intestinal or ureteric obstruction or hematuria. Per cutaneous intra-lesional Sclerotherapy is one of the options to reduce the size of lesion significantly. The radical surgical excision is still by far the treatment of choice with low recurrence rate and less complications.
\end{abstract}

Volume 9 Issue 3 - 2019

\section{Farida Ambusaidi, Latifa Al-Mammari, Yahya Al-Brashdi}

Pediatric Radiologist Specialist, Radiology Department lbri Hospital, Sultanate of Oman

Correspondence: Farida Ambusaidi , Pediatric radiologist
Specialist, Radiology Department Ibri Hospital, Sultanate of
Oman, Tel 00968997938I0, Email farida.ambusaidi@gmail.com

Received: May 20, 2019 | Published: May 28, 2019

\section{Introduction}

Lymphatic malformations are type of congenital vascular malformations. It is commonly seen in head and neck area. ${ }^{1,2}$ It can be seen in the abdomen in the liver, spleen, pancreas, mesentery and retroperitoneam. Retroperitoneal location is very rare with cases compromised less than $1 \%$ from all abdominal cases. ${ }^{1}$ Most of the patients are asymptomatic, however minority may present with abdominal distension or pain, intestinal or uretric obstruction or hematuria, cyst infection or hemorrhage. ${ }^{2-4}$ The cases usually present in the first two years of life and the initial investigation include ultrasound which shows multicystic lesion crossing multiple compartments. Retroperitoneal extension is better assessed by CT scan or MRI studies. There are multiple options to significantly reduce the size of lesion, including aspiration and Sclerotherapy, however both have high recurrence rate. ${ }^{1,5}$ The radical surgical excision is still by far the treatment of choice with low recurrence rate and less complications. ${ }^{1,4,5}$

\section{Case report}

We present 17 months old boy who was born as full term baby and discharged home in first 24 hours of life in good condition after routine neonatal examination which was unremarkable. He got trivial trauma. The family brought the child to the emergency department with alleged hit by car at very slow speed while his uncle getting car out from parking. The parents denied any history of loss of consciousness, vomiting, or seizure. The primary survey showed $9.8 \mathrm{~kg}$, conscious child who was crying vigorously with GCS $15 / 15$. His vitals were maintained: Oxygen saturation was $100 \%$ in room air, pulse 104 beat per minute. His airway was clear with equal air entry bilaterally. He had normal breathing pattern. He was hemodynamically stable. No focal neurological deficit. His abdomen was distended since long time before the trauma according to the mother, no tenderness or rigidity. Upper and lower limbs movement was unremarkable. The only positive finding was bruise in right foot but movement was preserved. His radiological work up revealed unremarkable chest and right foot radiographs. The ultrasound scan of abdomen (Figure 1) showed sizable retroperitoneal multi- loculated cystic lesions extending from renal area to the pelvis with innumerable septations and anechoic fluid content. It was abutting the lower pole of both kidneys and posterior aspect of urinary bladder. Sonographically, these imaging features were in favor of retroperitoneal lymphatic malformation. No trauma related abdominal organs injury or free fluid was seen. The child was referred to pediatric team to investigate further the incidental finding of fast scan. The child was doing well and asymptomatic during his stay in the hospital. Child was booked for CT scan of abdomen and pelvis due to unavailability of MRI in our centre. The CT scan (multi detector CT using a 64-detector row system; Siemens Somatom, Germany) of the abdomen and pelvis showed large size retroperitoneal cystic lesion measuring $10.8 \times 3.5 \times 5.7 \mathrm{~cm}$ in Transverse, AP and CC dimensions. It showed neither perceptible wall nor internal enhancement. No calcification or fatty components were seen. It insinuated between the retroperitoneal structures with no invasion. Liver, spleen, pancreas, adrenals and kidneys were unremarkable (Figure 2) (Figure 3). Similar to sonography findings, these imaging features were likely representing retroperitoneal lymphatic malformation/lymphangioma. Then, the patient was referred to tertiary hospital to be under care of pediatric surgery unit and where facility for ultrasound guided Sclerotherapy was available. Under ultrasound guidance, a 20 gauge needle was used to access the lymphangioma (Figure 4). Approximately $45 \mathrm{ml}$ of yellowish fluid was aspirated and then was sent to the laboratory for cytology which confirms the presence of high lymphocytes count. Following that, a $10 \mathrm{ml}$ of $3 \%$ sodium tetradecyl sulfate (STS) foam was injected into the collection and was kept in same location for approximately 10 minutes. Small amount of STS was aspirated and finally $10 \mathrm{mg}$ of Bleomycin was injected into the area. Pressure was applied to the same area for a few minutes. No immediate complications occurred during the procedure. The patient remained stable post procedure and discharged in good condition in the next day. Plan was given to the family that he will be admitted after 6 weeks for the second session. So after 6 weeks, he was readmitted for second session of Sclerotherapy. Pre procedural ultrasound showed significant reduction in the size of the previously known retroperitoneal fluid collection. Multiple attempts were made to access the residual lymphatic malformation failed and the procedure was aborted. He remained stable post the procedure, no complication occurred. He was discharged next day in good condition. The child had ultrasound follow up every six months to check the size of the lesion which remained stable over the last two years. The surgeon preferred to go with semi-invasive Sclerotherapy in this case and to preserve surgical excision for cases with confined lesion and good surgical outcome (i.e. not insinuating between bowel loops and not involving mesenteric vessels). 


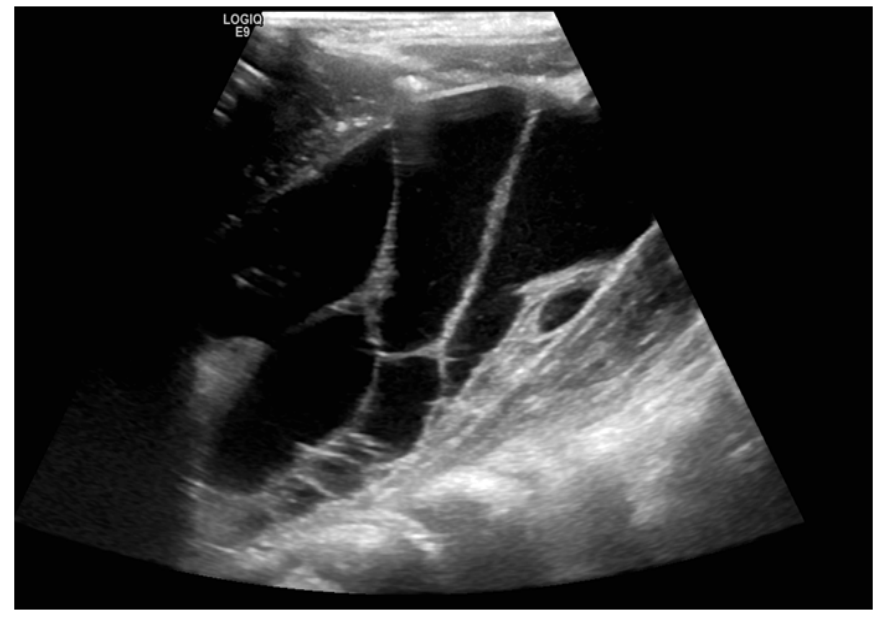

Figure I Retroperitoneal multi- loculated cystic lesions with innumerable septations and anechoic fluid content.
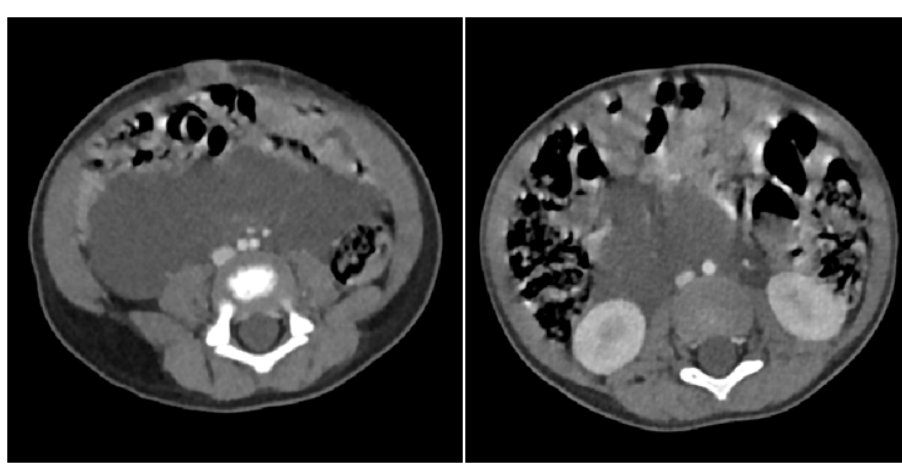

Figure $\mathbf{2}$ \& $\mathbf{3}$ shows large size retroperitoneal cystic lesion with neither perceptible wall nor internal enhancement. It insinuates between the retroperitoneal structures with no invasion.

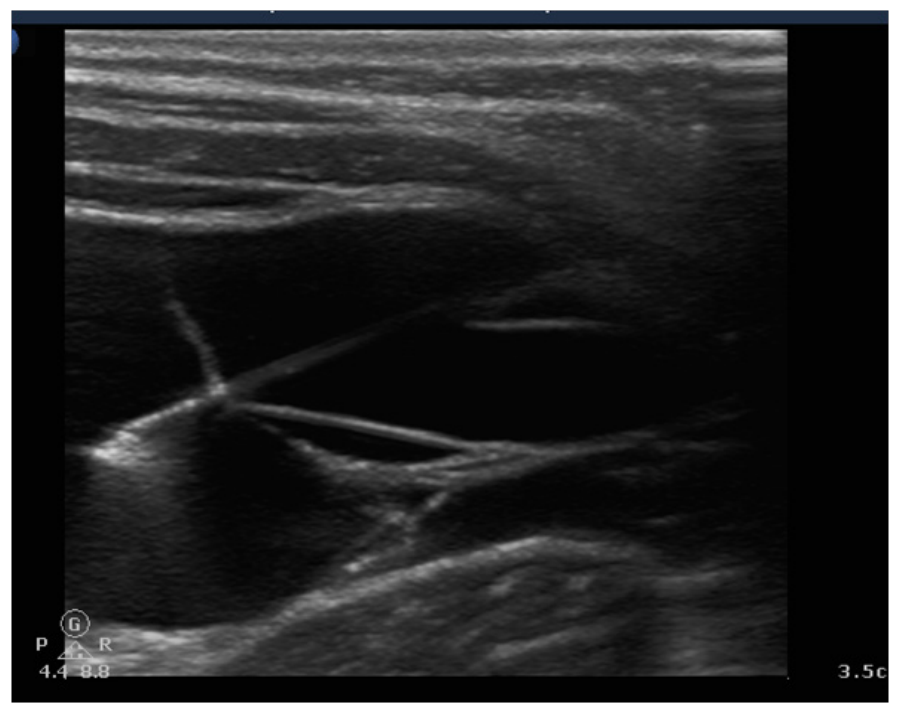

Figure 4 Under ultrasound guidance, a 20 gauge needle was used to access the lymphangioma.

\section{Discussion}

Lymphatic malformation is slow-flow vascular malformation. It is consistent of deformed cyst-like lymphatic channels which fail to drain into normal lymphatic chain. ${ }^{3}$ According to the size, it is divided into macrocystic, microcystic or mixed types cysts. ${ }^{2,5} \mathrm{We}$ present a case of macrocystic type where most of the cysts are larger than 1 $\mathrm{cm}$. Retroperitoneal location is rare and account for less than $1 \%$ of all abdominal lymphangioma. It can be seen in the peri-renal, para-renal and peri-vascular spaces and pelvic area. ${ }^{3}$ Children can present earlier with abdominal distension or pain. ${ }^{4}$ Majority of patient are asymptomatic and findings are incidental during scan for other reasons. ${ }^{6}$ Ultrasonography is the modality of choice in the initial assessment and evaluation of the morphology, location, vascularity of the lesion. It shows a vascular multiloculated lesion with septations and anechoic fluid content. ${ }^{2} \mathrm{CT}$ scan and MRI can be done to further assess the lesions extension and characteristics. ${ }^{3,5}$ CT scan may shows multicystic lesion with near water density and thin enhancing septations. The MRI features of lymphangioma are variable and include heterogeneous signal intensity of the macrocystic lesions predominantly low in $\mathrm{T} 1$ and high in $\mathrm{T} 2$ weighted images. There is sometimes fluid levels and blood components. , $3,5,6^{-3}$

The elongated shape, insinuating nature and the involvement of multiple compartments in this patient CT scan are important features which favor the diagnosis of lymphangioma over other differential. ${ }^{3}$ Aspiration of the fluid is helpful in establishing the diagnosis in our patient and yield the presence of milky fluid with high lymphocyte count. The tissue assessment post surgical excision is virtually diagnostic of lymphangioma and shows cystic spaces lined with a single thin layer of endothelium, lymphoid tissue and smooth muscles. ${ }^{4-8}$ However excision was deferred in this case due to involvement of mesenteric vessels and multiple compartments. Interventional radiology was opted to help in reducing the size of the lesions by attempting multiple Sclerotherapy sessions. They work by destroying lymphatic endothelial layer using intra-lesional sclerosing agents such as sodium tetradecyl sulfate (STS), bleomycin, doxycycline and ethanol. ${ }^{5}$ This child underwent dual-drug technique which gives excellent results with partial ablation. There are reported cases of complete ablation of lymphangioma utilizing dual-drug technique. ${ }^{5}$ It starts with the use of STS which cause pores formation in the cysts wall and increased efficiency of the second drug to destroyed the endothelial lining. ${ }^{5}$ Sometimes it is difficult to differentiate lymphatic malformation from other retroperitoneal cystic lesions, hence surgical excision and histopathology assessment are recommended. ${ }^{7,9}$ Also it is needed to reduce the complication like progressive increased in size, infection, intra-lesional bleed. ${ }^{9}$

\section{Conclusion}

Retroperitoneal lymphatic malformation should be considered in the differential diagnosis of elongated multicystic lesion insinuating through multiple compartments. Ultrasound is the modality of choice in initial assessment of the lesion followed by CT scan or MRI to delineate lesion extension. Surgical excision is the main stay treatment though semi-invasive Sclerotherapy can contribute significantly to pre-surgical size reduction and even to complete ablation.

\section{Acknowledgments}

None. 


\section{Conflicts of interest}

The authors declared there is no conflict of interest.

\section{References}

1. Lal A, Gupta P, Singhal M. Abdominal lymphatic malformation: Spectrum of imaging findings. Indian journal Radio. 2016;26(4):423428.

2. Michael LF, Candace LW, Brandon O, et al. Intraabdominal lymphatic malformations: Pearls and pitfalls of diagnosis and differential diagnoses in pediatric patients. Pediatric imaging. 2017;208(3):637-649.

3. Prabhakar R, Rakesh S, Carlos $\mathrm{C}$, et al. Imaging of uncommon retroperitoneal masses. Gatsrointestinal imaging. 2011;31(4).

4. M Di Marco, E Grassi, S Vecchiarelli, et al. Retroperitoneal lymphangioma: a report of 2 cases and a review of the literature regarding the differential diagnoses of retroperitoneal cystic masses. Oncol Lett. 2016;11(5):3161-3166.
5. William E Shiels II. Lymphatic Malformation: Radiologic-PathologicTherapeutic Correlation and Management Implications. J Am Osteopath Coll Radiol. 2012;1(3):2-8.

6. Calmes D Medart L. Abdominal lymphatic malformation. Journal of the Belgian society of radiology. 102(1):57.

7. Tripathi M, Parshad, Karwasra RK. Retroperitoneal lympangioma in adult: a case report of a rare clinical entity. Case Reports in Surgery. $2015 ; 732531$.

8. Yang DM, Jung DH, Kim H, et al. Retroperitoneal cystic masses: CT, clinical, and pathologic findings and literature review. Radiographics. 2004;24(5):1353-1365.

9. Bhavsar T, Saeed-Vafa D, Harbison S, et al. Retroperitoneal cystic lymphangioma an adult: a case report and review of the literature. The World Journal of Gastrointestinal Pathophysiology. 2010;1(5):171-176. 